This is a postprint version of the following published document: Ricardo Flores Moyano; David Fernández; Luis Bellido; Noemí Merayo; Juan Carlos Aguado; Ignacio de Miguel. NFV-based QoS provision for Software Defined Optical Access and residential networks. In: 2017 IEEE/ACM 25th International Symposium on Quality of Service (IWQoS). DOI: https://doi.org/10.1109/IWQoS.2017.7969142

(C) 2017 IEEE. Personal use of this material is permitted. Permission from IEEE must be obtained for all other uses, in any current or future media, including reprinting/republishing this material for advertising or promotional purposes, creating new collective works, for resale or redistribution to servers or lists, or reuse of any copyrighted component of this work in other works. 


\section{NFV-based QoS provision for Software Defined Optical Access and Residential Networks}

\author{
Ricardo Flores Moyano, David Fernández, Luis \\ Bellido \\ Departamento de Ingeniería de Sistemas Telemáticos \\ Universidad Politécnica de Madrid, Spain \\ \{rflores, david\}@dit.upm.es
}

\author{
Noemí Merayo, Juan Carlos Aguado, Ignacio de \\ Miguel \\ Grupo de Comunicaciones Ópticas \\ Universidad de Valladolid, Spain \\ \{noemer, jaguado, ignacio.miguel\}@ tel.uva.es
}

\begin{abstract}
The promises of SDN and NFV technologies to boost innovation and to reduce the time-to-market of new services is changing the way in which residential networks will be deployed, managed and maintained in the near future. New usercentric management models for residential networks combining SDN-based residential gateways and cloud technologies have already been proposed, providing flexibility and ease of deployment. Extending the scope of SDN technologies to optical access networks and bringing cloud technologies to the edge of the network enable the creation of advanced residential networks in which complex service function chains can be created to provide traffic differentiation. In this context, this paper defines a new network management model, based on a user-centric approach, that allows residential users to define and control access network resources and the dynamic provision of traffic differentiation to fulfill $Q o S$ requirements.
\end{abstract}

Keywords-NFV; SDN; QoS; Edge Cloud; traffic differentiation

\section{INTRODUCTION}

The provision of Internet services for residential environments or small offices is changing due to the gradual adoption of Software Defined Networks (SDN), Network Functions Virtualization (NFV) and Cloud technologies by the Internet Service Provider (ISP). As mentioned in [1], a Software Defined Access Network is intended to abstract the entire access network by means of a common and unified interface for management and control. This approach enables the agile creation of new services adapted to the user needs. Considering that optical transmission media is becoming the preferred option for access networks, the objective is to make the access node or Optical Line Terminal (OLT) compliant with the SDN principles. Nowadays, SDN-based OLTs that natively implement OpenFlow are not commonly available. A hardware abstraction layer is used to turn legacy OLTs into OpenFlow-controllable devices [2]. Different proposals are aligned with this approach. For instance, an OpenFlow-enabled Gigabit-capable Passive Optical Network (GPON) is presented in [3]. The hardware abstraction layer presents to the external OpenFlow controller a single distributed switch with several

Ricardo Flores Moyano is supported by SENESCYT, Ecuador through a scholarship program (2013-AR6C548). This research has been partially supported by the Spanish Ministry of Economy and Competitiveness in the context of GREDOS project (TEC2015-67834-R), TEC2014-53071-C3-2P and Elastic Networks (TEC2015-71932- REDT). ports, one for the OLT and the remaining ports for each Optical Network Terminal (ONT). A similar proposal is presented in [4] where an embedded OpenFlow agent resides within the OLT. The agent interacts with the external OpenFlow controller and the GPON OMCI (ONT Management and Control Interface) module, transforming the entire GPON system into a virtual switch.

With the aim of providing a great flexibility to facilitate the development of new and better services for residential networks, the virtualization of the Residential Gateway (RGW) is envisioned. The home network virtualization use case proposed by ETSI [5] and the Network Enhanced Residential Gateway (NERG) architecture proposed by the Broadband Forum [6] are representative examples of this approach. An L2-device replaces the traditional RGW and the remaining network functions like routing or Network Address Translation (NAT) are implemented as virtual instances at the ISP's NFV Infrastructure (NFVI). Although the vRGW (virtual RGW) approach leverages the elasticity and agility of cloud technologies, the NVFI must be properly dimensioned to support all the customers' demands while assuring an adequate level of Quality of Service (QoS). In this light, the Edge Cloud [7] or Edge Computing [8] represents a novel alternative to centralized datacenters located at the ISP's core network. An Edge Cloud provides lower latency, reduces the backbone traffic and provides an initial stage for traffic treatment. A NFVI deployed at the edge not only allows instantiating traditional network functions like routing or NAT but also creating complex Service Function Chains (SFCs) to enrich the traffic treatment of residential networks. The TR-345 of Broadband Forum [9] implements a NFVI to augment or replace the services provided by the Broadband Network Gateway (BNG) with Virtualized Network Functions (VNFs). In this scenario, the subscriber traffic is selectively steered to additional service functions deployed at the NFVI. An SFC could be applied to all subscriber traffic or specific flows.

The Central Office Re-architected as a Data Center (CORD) initiative [10] joins together the SDN, NFV and Edge Cloud paradigms to transform the legacy Central Office. CORD significantly contributes in proposing a unified platform to improve the provision of telecommunication services. The Residential CORD proposal includes a Software Defined Optical Access Network (SDOAN) composed of an OpenFlow-enabled OLT and its software counterpart appointed as virtual OLT (vOLT). Similar to ETSI and Broadband Forum, CORD virtualizes the RGW, placing a L2 device at the user residence and deploying a virtual Subscriber Gateway 
(vSG) at the Edge Cloud. All the Residential CORD components are orchestrated by a centralized management platform.

The analysis of the state of the art shows a clear consensus regarding the virtualization of the RGW. However, considering the limitations of an L2-device, an SDN-based RGW would enable a fine-grained control of the traffic. The TR-317 is focused on showing the benefits of NERG and specifies some requirements to achieve them. However, it lacks a detailed description on how to add new network functions to the virtual gateway (vG). The TR-345 in a way tries to support the NERG architecture advancing in the description of SFC in alignment with the NFV paradigm. However, the Broadband Forum requires a further specification to integrate the access network as well as the Edge Cloud paradigm in its proposals. CORD goes beyond the Broadband Forum proposals and presents a unified platform for ISPs. However, CORD lacks a detailed description on how to create a customized SFC per traffic flow for a given residential network. In addition, none of the presented proposals consider the possibility to engage the residential user in the management tasks of their own networks. In this work in progress paper, the CORD vision is further developed to provide the residential user the ability to manage its own network, defining customized SFCs in charge of providing a differentiated traffic treatment with QoS.

\section{A USER-CENTRIC MANAGEMENT APPROACH}

Considering that a certain level of technical expertise is required to manage a residential network, a user-centric approach as mentioned in [11] represents a significant improvement in this, sometimes, frustrating activity. Based on the requirements and skills of residential users, a Residential Management application (RENEMA app) is conceived to involve them in the management tasks of their own networks. Taking into account the high flexibility and programmability of the SDN technologies, a RENEMA app resides on top of the SDN reference architecture in order to provide a usercentric management architecture as depicts Fig. 1. With the aim of simplifying the creation of applications different Residential Network Services (RENESEs) are provided, each one providing a specific functionality with a certain abstraction level that hides the complexity of the network configuration. Network Resources Control (NERON) and Network Statistics (NESA) represent additional functionalities to allocate network resources and to provide network statistics respectively. Thereby, an application represents an advanced service composed of different RENESEs in charge of improving certain aspects of residential networks like the Ease of Management, Visibility, QoS or Security. The user requirements are captured at the Interaction layer and then are processed at the Management layer. Based on the configuration to be performed, the RENEMA app interfaces with different RENESEs. Finally, the corresponding flow entries are delivered to the Control layer.

The user-centric approach implements an SDN-based RGW to overcome the constraints of an L2-device and also follows the vRGW proposal. However, this approach could be significantly improved by including the SDOAN and Edge Cloud principles in the proposal. The resulting environment enables the residential user to also control the characteristics of the access network. Similarly, different SFCs per traffic class can also be defined at the Edge Cloud to enrich the traffic treatment according to the user needs.

\section{A COMPREHENSIVE MANAGEMENT SYSTEM}

In this work in progress paper, the user-centric approach is extended. The User-centric Management System is in charge of orchestrating the SDOAN and Edge Cloud (NFVI) extra components in order to provide a comprehensive and centralized management platform. As Fig. 2 depicts, the objective is to provide a customizable virtual slice of access network per residential network. On the SDOAN Infrastructure (SDOANI), a virtual slice is instantiated and the SDOAN's control system exports an API through the $A N-U C$ (Access Network - User-Centric Management System) interface to manage the functional parameters of the virtual slice.

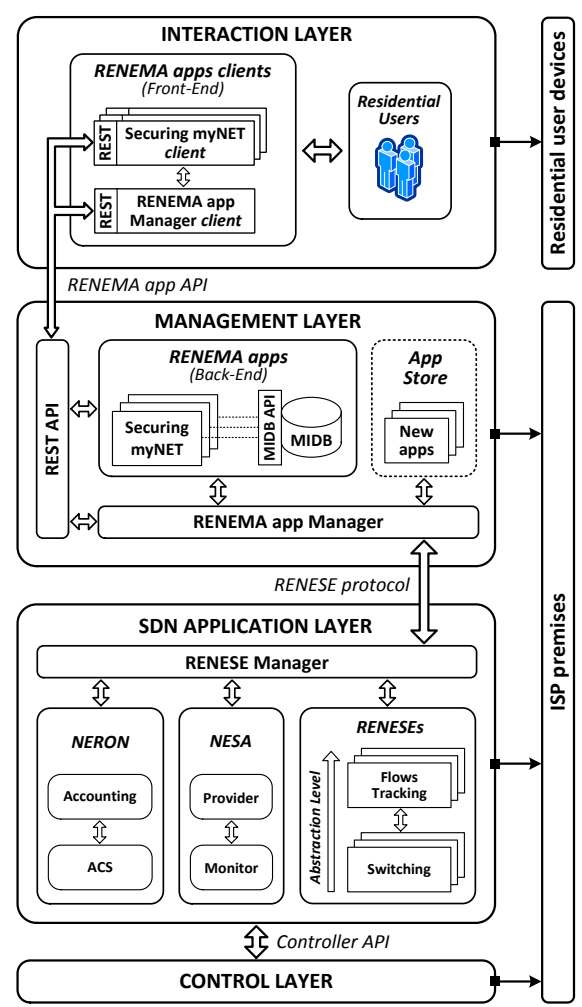

Fig. 1. User-centric management approach. Additional RENEMA apps are in charge of interfacing with the SDOAN's control and the NFV Management and Orchestration (MANO).

The user-centric management system captures the user requirements and submits them to the SDOAN using the corresponding API.

By means of the SDOAN's API a number of parameters could be adjusted in order to configure the virtual slice of the access network, such as the minimum guaranteed bandwidth levels in both the upstream and downstream channel, delay and jitter guarantees in the access network, and maximum packet loss rate, all while taking into account traffic prioritization or classification. An example of the functionalities offered by such an API will be later presented in Section IV.

From the management point of view, a RENEMA app in charge of managing the functional parameters of the virtual access network slice must be incorporated to the user-centric management system. This application implements the $A N-U C$ interface to use the functionalities provide by the SDOAN's control API mentioned above.

Following the trend of bridging the Cloud to the edge, a NFV infrastructure is co-located with the OLT at the Central Office. This cloud infrastructure is in charge of hosting the virtualized Management and Networking Domain (vMANDO) which includes the management system instance and the outsourced virtualized network functions of the residential 
network. As Fig. 2 depicts, each virtual network slice is connected to a vMANDO through the $A N-C L$ (Access Network - CLoud) interface. Considering the physical and logical placement of the different SDOANI and NFVI components, the $A N-C L$ interface is implemented by means of physical and virtual networks to achieve the required functionality.

With the aim of enriching the differentiated traffic treatment with QoS, a network segmentation schema for the residential network is proposed. The residential user is able to define different network segments, each one with a specific traffic class.

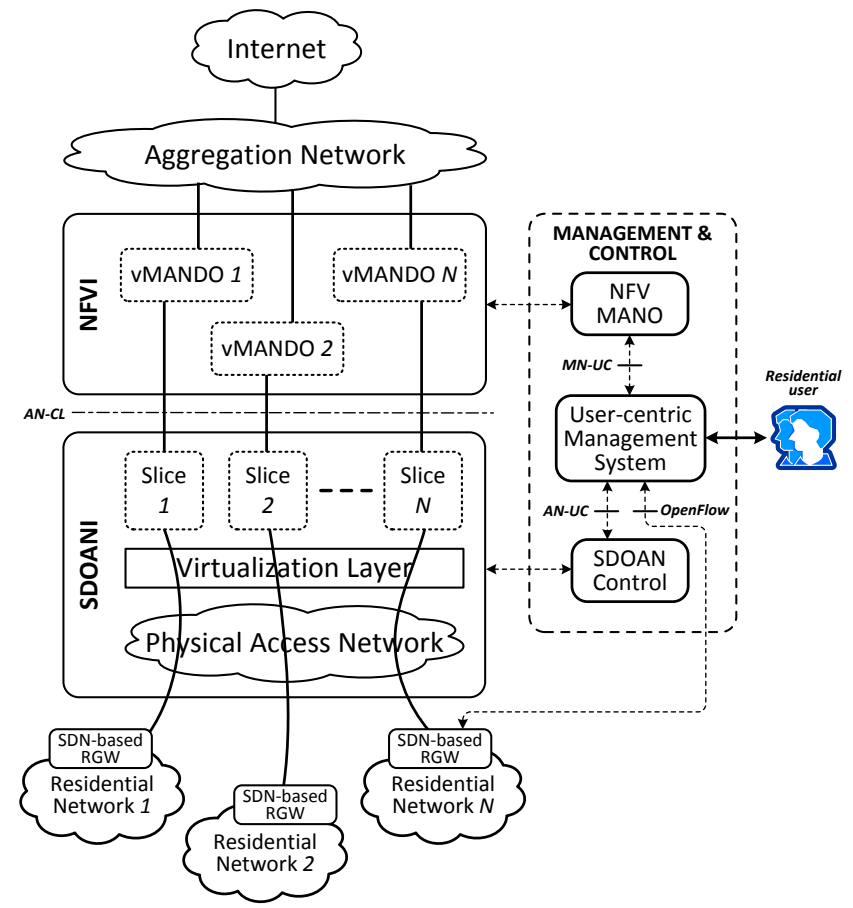

Fig. 2. The virtual network slice is connected to its corresponding vMANDO at the NFVI. The user-centric management system interfaces with the SDOAN's control system and the NFV MANO to manage the different components of this new residential network ecosystem.

Since there is an SDN-based RGW, the network segments could be defined by means of, for example, physical ports, device's MAC address, device's IP address or TCP/UDP ports. The residential user also might define a Service Function Chain per network segment. Multiple network segments and SFCs require the specification of an ID to identify a specific network segment and its corresponding SFC at the vMANDO.

Similarly, this ID is used to also define a virtual path to properly steer the traffic from the residential network to the SFC. Different options to differentiate the network segments could be used, for instance: VLANs, VxLAN, tunnels or Network Service Header (NSH). To better explain the proposal, a VLAN-based network segmentation approach as mentioned in [12] is embraced.

The residential network traffic is double-tagged using the 802.1 ad standard (Q-in-Q). The outer S-tag allows identifying the residential network and the inner $\mathrm{C}$-tag allows identifying the network segment. For instance, the residential user might define different network segments to associate them to a specific type of traffic as depicted in Fig. 3. The Q-in-Q mechanism is also embraced by CORD. However, as the multiple SFCs per residential network approach is not considered, its implementation is different. The S-tag identifies the virtual machine where the container-based $\mathrm{vSG}$ resides and the $\mathrm{C}$-tag identifies the container itself.

Based on the S-tag the Delivering network steers the traffic of a given residential network to its corresponding vMANDO as depicted in Fig. 4. Once there, the residential network traffic requires two levels of classification due to the in-band control of the SDN-based RGW. The first level is in charge of classifying the control traffic and the data traffic (Classification network) whereas the second level is in charge of classifying the traffic class associated to a network segment (Classification \& Shaping). The Classification and Delivering networks are implemented using for example Open vSwitch (OVS). These networks could be merged into a single network in charge of classifying and steering the traffic.

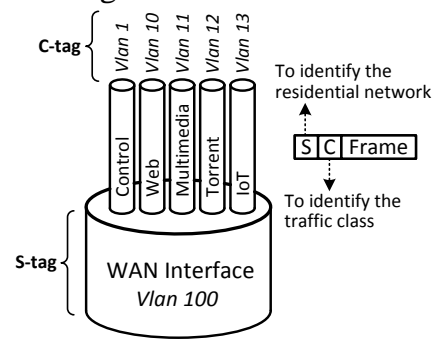

Fig. 3. By means of VLAN tags, the WAN interface is divided in multiple virtual channels in charge of carrying the traffic of a network segment to its corresponding SFC.

However, an approach like this implies that the mechanism to segment the residential network would have a global meaning. In the VLAN case, the 12-bits VLAN ID field results in a limited number of VLANs available to all ISP's customers. Moreover, a single-network approach also requires an effective mechanism to implement the two-level hierarchical identification for the different network segments defined per residential network, which could result in a complex task due to security concerns.

The management and networking components are instantiated following the residential user requirements within the vMANDO at the NFVI. The vMANDO's networking component is composed of different VNFs. Router + NAT and Classification \& Shaping are fixed VNFs that must be mandatorily deployed per residential network in order to provide a basic or default service. Considering that the WAN interface of the SDN-based RGW is divided into several virtual channels to carry the traffic of a network segment to its corresponding SFC, the rate limiting and traffic classification functions of the Classification \& Shaping VNF are used to create the required number of virtual channels terminations. Following an on-demand model, between the two fixed VNFs, different VNFs might be connected through a dynamic virtual network to create a specific SFC. This approach provides a great flexibility to apply different treatment strategies to a given network segment, enriching the current QoS features provided by the SDOAN. For instance, the residential user might define two network segments to differentiate the multimedia traffic from the web traffic as depicted in Fig. 5. Similarly, two SFCs are also defined based on the available VNFs and associated to the corresponding network segments. A WAN accelerator (WANA) is added to the segment A (multimedia) whereas a Content Filter and a Firewall are added to the segment B (Web). In a more advanced scenario, a Deep Packet Inspection (DPI) function could be added in order to recognize suspicious traffic patterns.

In this new residential network environment configuration, the SDN-based RGW, the SDOAN and the Classification \& Shaping VNF lay down a three-tier rate limiting policies definition mechanism for the virtual path created between the residential network and the distribution network. Each level enables the definition of queues for both, upstream and downstream traffic. These policies are intended to improve the QoS of specific services provided by the ISP like VoD, IPTV or voice. For instance, the residential user could define a QoS 
policy to watch a specific TV show. Based on the defined QoS policy, the user-centric management system modifies the rate limiting policies of the three tiers. If the QoS resulting is not the expected one, a Bandwidth on Demand (BoD) approach could be implemented by the SDOAN. The user specifies the additional bandwidth value and depending on the periodicity of the event a time schedule is also specified.

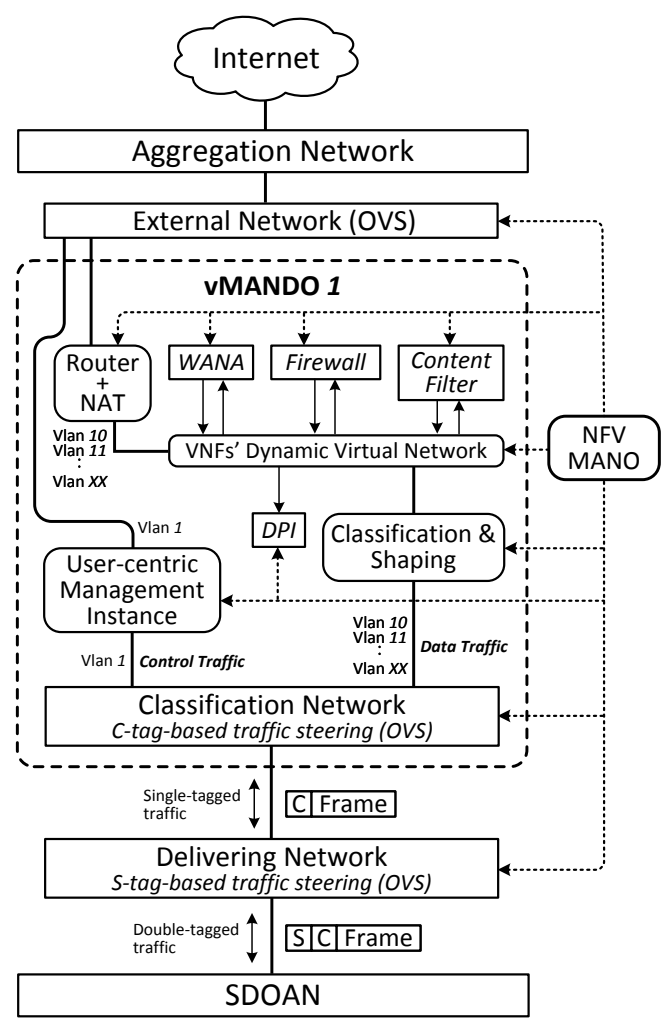

Fig. 4. NFV MANO is in charge of instantiating different VNFs to create a SFC according to the specifications received from the user-centric management system.

The NFV Management and Orchestration (MANO) provides the functionality required for the provisioning of VNFs, and related operations such as the configuration of VNFs and the infrastructure on which these functions run [13]. According to Fig. 2, the NFV MANO's services or functionalities should be accessible through the $M N-U C$ (MANO - User-Centric Management System) interface in order to enable the residential user to configure specific SFCs.

Following this approach, a RENEMA app composed of a function to segment the residential network (Network Segment Definer) and a function to create an SFC (SFC Creator) must be incorporated to the User-centric management system. This application implements the $M N-U C$ interface to use the NFV MANO's API. Depending on the mechanism used to segment the network, the Network Segment Definer coordinates the creation of the network segment on the SDN-based RGW and the virtual path for its corresponding SFC. For the VLAN case, the Switching service running at the SDN application layer is instructed on how to tag the traffic based on the network segments previously defined. At the NFVI, the NFV MANO is also instructed on how to install the required flow rules to classify and steer the traffic to the appropriate vMANDO. Moreover, the SFC Creator retrieves the VNFs catalog from the NFV MANO in order to enable the residential user to create and associate an SFC to a specific network segment.

Considering that the proposal is aligned with the NFV Architectural framework proposed by ETSI [14], different initiatives in charge of implementing the NFV MANO [13] might be used. However, the SFC API provided by the NFV platform should be extended in order to meet the requirements regarding the creation of virtual paths (Delivering and Classification networks and Classification \& Shaping VNF) and their corresponding SFCs (VNFs' Dynamic Virtual Network).

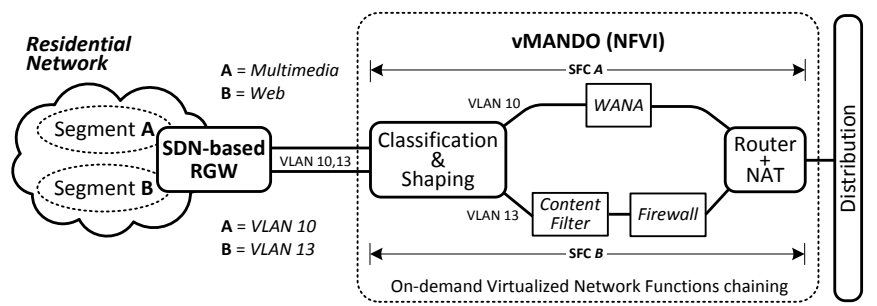

Fig. 5. SFC $A$ and SFC $B$ have been defined for segments $A$ and $B$ respectively within the residential network for differentiated traffic treatment with QoS.

For instance, as mentioned in [15], to create a service chain a dataset containing a service description as an ordered sequence of VNFs' connection points is passed to Netfloc ${ }^{1}$ by invoking the Create chain REST API. In addition to describing the SFC by itself, this dataset should contain additional descriptors to specify the required virtual paths to steer the traffic to the corresponding SFCs based on the network segments defined. Besides the option to create an SFC, the API should also provide additional options to enhance the residential user experience. For instance, an API to query the status of the different SFCs, the computational resources being consumed, the provision of templates for creating basic or advanced SFCs or even the possibility to share an SFC to other residential users.

\section{EXPERIMENTAL TESTBED}

With the aim of validating the proposal, a testbed composed of the Access Network, the Edge Cloud and the User-centric management system is being implemented. The testbed is based on the proof of concept scenario developed to validate the user-centric approach [11]. Considering that OpenStack already provides the NFVI and the corresponding Virtualized Infrastructure Manager (VIM), the remaining MANO components like the NFV Orchestrator and the VNF Manager [14] should be added to enable the creation of SFCs. As the SDOAN is being implemented from scratch, a detailed explanation is provided in this work in progress paper.

The SDOAN is composed of a GPON facility (left in Fig. 6) and the corresponding control module explained below. The GPON is implemented using equipment from the Telnet-RI vendor $^{2}$. In particular, we use an OLT SmartOLT 350, which implements a full-duplex GPON interface of $2.488 \mathrm{Gbps}$ (downstream) and 1.244 Gbps (upstream), being fully compliant with ITU-T G.984.1-4 and G.988 specifications. It includes 4 ports, each supporting up to 64 ONTs. Moreover, it also complies with 802.1q and 802.ad standards, thus enabling $\mathrm{C}$ and $\mathrm{S}$ tagging as previously described in Section III. Furthermore, the connection between the OLT and the optical splitter includes three spools of Standard Single Mode Fiber (SSMF) of different lengths (two of $5 \mathrm{~km}$ and one of $10 \mathrm{~km}$ ), thereby permitting to configure the reach of the network to 5, 10,15 or $20 \mathrm{~km}$ (plus the length of the distribution fiber, as later described). The testbed is also equipped with two optical splitters 1:8, thus enabling the configuration of a two-stage splitting topology if desired. Then, the splitters are connected to the ONTs by means of distribution fibers. The length of each link can be individually configured, by means of a connection

\footnotetext{
${ }^{1}$ https://github.com/icclab/netfloc

${ }^{2} \mathrm{http}: / /$ www.telnet-ri.es/en
} 
panel, from 100 meters up to $5 \mathrm{~km}$. In this way, the testbed can be used to analyze realistic scenarios where different users have different delays from their location to the central office. Finally, at this moment, the testbed employs L3 model ONTs (Wave Access 3021), which means that they integrate router functionalities.
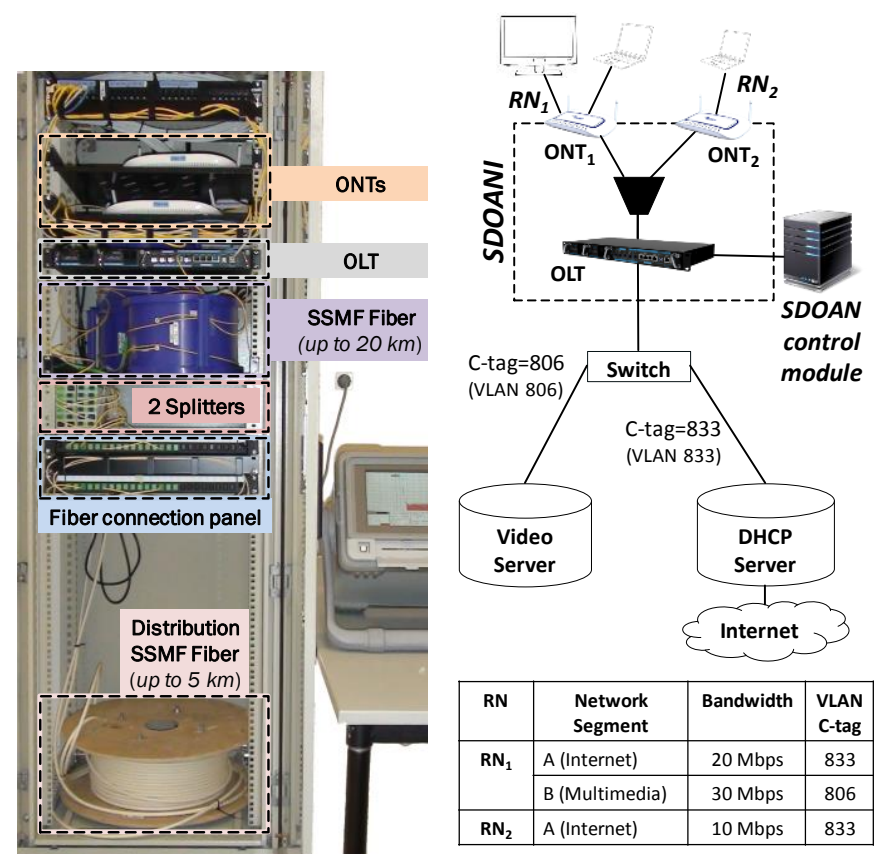

Fig. 6. (Left) Real deployment and configuration of the GPON testbed. (Right) Example of an initial test configuration in the GPON testbed.

Those ONTs comply with the ITU-T G.984.x and G.988 specifications, supporting 2.488 Gbps (downstream) and 1.244 Gbps (upstream).

The configuration of different services (in particular, Internet and multimedia services), as well as user (or residential network) profiles in the testbed can be done by means of two management control interfaces offered by TelnetRI equipment. The first one is the TGMS (TELNET GPON Management System), a visual web platform that permits to manage and to configure the OLT and the connected ONUs. The second is a Command Line Interface (CLI). The SDOAN control module, is currently being developed in Python, using the CLI, and automatizing the management of the optical access network testbed. At this stage of development, the SDOAN control module offers the following functionalities:

- Initialization, load, discard and visualization of the configuration of the GPON network (OLT, ONTs, and parametrized network segments).

- Configuration and modification of Internet services, by setting a number of parameters:

- Downstream guaranteed and excess bandwidth

- Upstream guaranteed and excess bandwidth.

- VLAN identifier (C-tag) associated with the service.

- Configuration and modification of multimedia (video) services, by setting the same parameters mentioned above.

- Configuration of residential network profiles integrating Internet and multimedia network segments.

An example of an initial test configuration of the GPON testbed is shown in Fig. 6 (right). Two different residential networks $\left(R N_{1}, R N_{2}\right)$ have been created. The first one, $R N_{l}$, has defined two network segments, Internet, setting the bandwidth parameter to $20 \mathrm{Mbps}$, and multimedia (video) running at 30 Mbps. In order to differentiate those segments, C-tagging is used, in particular C-tag 833 is used for the Internet segment and 806 for the multimedia service. The second residential network, $R N_{2}$, has defined a single network segment, for Internet, thus sharing the $833 \mathrm{C}$-tag, but setting the bandwidth to $10 \mathrm{Mbps}$. The Python-based SDOAN control module has been used for setting up such a configuration, and successful operation of the network in those conditions have been demonstrated.

\section{CONCLUSIONS}

This work in progress paper has presented the enhancement of a user-centric management proposal for residential networks with the functionality to control the SDOAN and to create SFCs per traffic class to enrich the QoS provision. This approach remarkably increases the flexibility to create bespoke residential networks, according to user requirements.

An initial experimental testbed to validate the proposal is currently being developed, with a particular attention in the SDOAN. A GPON facility has been implemented together with an initial prototype of the SDOAN control module, whose operation for defining and operating different networks segments (for Internet and video) has been validated.

Besides, the present work is focused on the development of a hardware abstraction layer for the SDOAN, as well as the integration of all the elements of the architecture presented. Special attention is being paid to the further specification and definition of the standard APIs needed to orchestrate all the components involved in the architecture.

\section{REFERENCES}

[1] K. J. Kerpez, J. M. Cioffi, G. Ginis, M. Goldburg, S. Galli, and P. Silverman, "Software-defined access networks," IEEE Commun. Mag., vol. 52, no. 9, pp. 152-159, 2014.

[2] A. S. Thyagaturu, A. Mercian, M. P. McGarry, M. Reisslein, and W. Kellerer, "Software defined optical networks (SDONs): A comprehensive survey," IEEE Commun. Surv. Tutorials, vol. 18, no. 4, pp. 2738-2786, 2016.

[3] R. G. Clegg et al., "Pushing software defined networking to the access," in Software Defined Networks (EWSDN), 2014 Third European Workshop on, 2014, pp. 31-36.

[4] S. S. W. Lee, K.-Y. Li, and M.-S. Wu, "Design and Implementation of a GPON-Based Virtual OpenFlow-Enabled SDN Switch," J. Light. Technol., vol. 34, no. 10, pp. 2552-2561, 2016.

[5] ETSI, "Network Functions Virtualisation, Use Cases (V1.1.1)," 2013. [Online]. Available: https://goo.gl/1HrZlw. [Accessed: 27-Feb-2017].

[6] Broadband Forum, "TR-317 Network Enhanced Residential Gateway," 2016. [Online]. Available: https://goo.gl/n03nth. [Accessed: 27-Feb2017].

[7] H. Chang, A. Hari, S. Mukherjee, and T. V Lakshman, "Bringing the cloud to the edge," in Computer Communications Workshops (INFOCOM WKSHPS), 2014 IEEE Conference on, 2014, pp. 346-351.

[8] W. Shi and S. Dustdar, "The promise of edge computing," Computer (Long. Beach. Calif)., vol. 49, no. 5, pp. 78-81, 2016.

[9] Broadband Forum, "TR-345 Broadband Network Gateway and Network Function Virtualization," 2016. [Online]. Available: https://goo.g1/RAF0rw. [Accessed: 27-Feb-2017].

[10] L. Peterson et al., "Central office re-architected as a data center," IEEE Commun. Mag., vol. 54, no. 10, pp. 96-101, 2016.

[11] R. Flores Moyano, D. Fernández Cambronero, and L. Bellido Triana, “A user-centric SDN management architecture for NFV-based residential networks," Comput. Stand. Interfaces, 2017.

[12] E.-D. Kim, S. Lee, Y. Choi, M.-K. Shin, and H.-J. Kim, "Evaluations of a multiple flow tables construction scheme for service function chaining," in Information Networking (ICOIN), 2016 International Conference on, 2016, pp. 424-426.

[13] R. Mijumbi, J. Serrat, J.-L. Gorricho, S. Latré, M. Charalambides, and D. Lopez, "Management and Orchestration Challenges in Network Functions Virtualization," IEEE Commun. Mag., vol. 54, no. 1, pp. 98$105,2016$. 
[14] ETSI, "Network Functions Virtualisation (NFV) Architectural Framework (v1.2.1)." [Online]. Available: https://goo.gl/jU3JDC. [Accessed: 27-Feb-2017].
[15] I. Trajkovska et al., "SDN-based service function chaining mechanism and service prototype implementation in NFV scenario," Comput. Stand. Interfaces, 2017. 